Sex differences in communicative gestures and speech comprehension: an ERP study

\title{
Mirella Manfredia ${ }^{a}$ Paulo Sergio Boggio ${ }^{b}$
}

aDepartment of Psychology, University of Zurich, Zurich, Switzerland; Social and Cognitive Neuroscience Laboratory, Center for Biological Science and Health, Mackenzie Presbyterian University, São Paulo, Brazil

\section{*Correspondence:}

Dr. Mirella Manfredi

Developmental Psychology: Infancy and Childhood

University of Zurich

Binzmuehlestrasse 14, 8050 Zurich, Switzerland.

mirella.manfredi@uzh.ch 


\begin{abstract}
The goal of this study was to investigate whether the semantic processing of the audiovisual combination of communicative gestures with speech differs between men and women. We recorded event-related brain potentials in women and men during the presentation of communicative gestures that were either congruent or incongruent with the speech. Our results showed that incongruent gestures elicited an N400 effect over frontal sites compared to the congruent ones in both groups. Moreover, only females showed an earlier N2 response to incongruent stimuli than congruent ones, while larger sustained negativity and late positivity in response to incongruent stimuli was observed only in males. These results suggest that women rapidly recognize and process audiovisual combinations of communicative gestures and speech (as early as $300 \mathrm{~ms}$ ) whereas men analysis at the later stages of the process.

Keywords: Event-related potentials; N400; N2; Gestures; Speech; Sex differences
\end{abstract}




\section{Introduction}

During the speech, speakers automatically support the speech with hand, face, and body gestures (Ozyurek, 2014). Kendon (1980) defines gestures as visible actions of the hand, body and face that are intentionally used to communicate and express themselves together with verbal information. As Ozyurek points out (2014), co-speech gestures can show different levels of complexity (e.g. pointing, representation of objects/events), and may have different functions both at the communicative level (e.g. emphasis, iconic) and in their relationship with speech.

In the present study, we used electrophysiological measures to examine whether the semantic processing of the audiovisual combination of communicative gestures with speech differs between men and women.

To investigate semantic processing, previous studies have measured event-related brain potentials (ERP), particularly focusing on the N400, an ERP component thought to index semantic processing. This ERP component peaks roughly $400 \mathrm{~ms}$ after the onset of a stimulus and is thought to index the spreading activation that occurs in the access of semantic information by a stimulus in relation to its preceding context (Kutas \& Federmeier, 2011). The amplitude of this component is greater to critical words that are semantically unrelated (vs. related) to previous single words in semantic priming paradigms (Bentin et al., 1985; Kutas \& Hillyard, 1980). The N400 also appears in semantic processing outside of language, but both the morphology and the latency of the N400 response seem to be sensitive to differences in sensory modality (Manfredi et al., 2018). The N400 also occurs in response to individual images (Van Berkum et al., 2003; Barrett \& Rugg, 1990) visual sequences (Sitnikova et al., 2008; Sitnikova et al., 2003; West \& Holcomb, 2002) or visual narratives like comics (Cohn, 2012). However, visual information typically elicits a more widespread frontal distribution 
(Barrett \& Rugg, 1990; Ganis et al., 1996; McPherson \& Holcomb, 1999) compared to written words (Kutas \& Hillyard, 1980).

The N400 also appears even across interactions between sensory modalities (i.e., vision and sound): previous works observed N400 effects when speech and/or natural sounds were combined with semantically inconsistent pictures or video frames (Cummings et al., 2008; Liu et al., 2011; Plante et al., 2000; Puce et al., 2007) or visual narrative (Manfredi et al., 2018; Manfredi et al., under review). Similar results occurred when gestures were combined with (in)congruent verbal information (Cornejo et al., 2009; Coulson \& Wu, 2005; Coulson \& Wu, 2005; Wu \& Coulson, 2007; Holle \& Gunter, 2007; Özyürek et al., 2007; Habets et al., 2011; Kelly et al., 2010; Proverbio et al., 2014a; Proverbio et al., 2014b). In 2007, Holle \& Gunter performed an ERP study in which they investigated whether iconic gestures could disambiguate the meaning of ambiguous speech. The EEG was recorded during the presentation of videos showing a person gesturing and speaking simultaneously. Auditory sentences contained a homonym in the initial part of the sentence that was disambiguated at a target word in the subsequent clause. Simultaneous with the homonym, the speaker produced an iconic gesture that was congruent or incongruent with the subordinate meaning. The results showed a smaller N400 effect to congruent gestures and larger in response to incongruent gestures, suggesting that participants used gestures to disambiguate speech. More recently, Proverbio and colleagues (2015) examined the electrophysiological responses associated with gesture comprehension preceded by a congruent or incongruent verbal description. Pictures of actors showing common Italian gestures were presented to healthy observers. The results revealed a great anterior $\mathrm{N} 400$ response to mismatch gestures.

Overall, these previous studies suggest that healthy individuals are able to integrate verbal information and communicative gestures into a coherent and integrated semantic representation. In the present study, we investigated whether the gender of observers affects 
the semantic processing of the simultaneous presentation of communicative gesture and speech.

Previous studies suggested that women are more able to recognize and process social information compared to men (Proverbio et al., 2017). For example, several works have shown that women have a more refined ability to interpret emotions through facial expression (Proverbio, 2017) and action purpose (Proverbio et al., 2010), compared with men. In previous research, Proverbio and colleagues (2010) investigated the semantic processing of congruent and incongruent actions in men and women. The results revealed greater N200 and N400 responses to incongruent actions in women compared to men, perhaps reflecting a difference in the biological relevance of unexpected social information in the two sexes.

Moreover, other studies suggested that the gender of observers also affects body language reading (Anderson et al., 2013; Pavlova, 2017; Krüger et al., 2013). For example, Sokolov and colleagues (2011), performed a study in which female and male observers had to recognize emotions through point-light human locomotion performed by actors with different emotional expressions. This study revealed suggested that males better recognized happy walking represented by female actors, while females better recognized angry locomotion expressed by male actors. In 2013, Anderson and colleagues carried out an fMRI study in which they presented point-light displays of coherent and scrambled biological motion in women and men. Their results revealed an enhanced activity during coherent biological motion perception in females compared to males in brain regions involved in social perception (e.g. amygdala, medial temporal gyrus, and temporal pole). Therefore, some previous studies showed gender differences in the ability to recognize and interpret the meaning of body language and suggested that women might be overall more sensitive to social stimuli than men (Proverbio et al., 2010; Proverbio et al., 2006; Proverbio, Zani, \& Adorni, 2008; Schirmer et al., 2008; Schirmer et al., 2005). 
Given both the observed differences in the ability to recognize and interpret facial expression, action goal and bodylanguge between women and men (Proverbio, 2017; Pavlova, 2017) and the enormous complexity of the mimicry and gestures that humans use to communicate, we believe that would be woth investigating the semantic processing associated with communicative co-speech gestures in men and women.

Thus, in this study we investigated whether the semantic processing of the audiovisual combination of communicative gestures with auditory information would differ between the two sexes. To this aim, several communicative gestures associated with semantically congruent or incongruent speech were presented to healthy men and women while recording their EEG response. Based on the previous findings (Sokolov et al., 2011; Anderson, 2013; Proverbio et al., 2010), we expected that if women are more sensitive to social information, (in)congruent co-speech gestures would elicit different ERPs responses (i.e. N2, N400) in women compared to men.

\section{Methods}

\section{Participants}

Thirty undergraduate students (15 females) were recruited from the Presbyterian Mackenzie University of São Paulo. Participants were native Portuguese speakers (mean age = 23.6, SE = 4.7), had normal or corrected-to-normal vision, and reported no history of neurological illness or drug abuse.

Their handedness was assessed by the Portuguese version of the Edinburgh Handedness Inventory (Oldfield, 1971), a laterality preference questionnaire reporting right-handedness dominance for all participants. Six participants were excluded from the ERP statistical analyses because of EEG artifacts, including excessive blinking, eye-movements, and muscular movements. This resulted in 24 final participants (13 females). The final sample size was 
similar to the one used by Proverbio and colleagues in the aforementioned study (Proverbio et al., 2009). The study adhered to the Declaration of Helsinki guidelines and was approved by the institutional ethics committee of Mackenzie Presbyterian University, Brazil, and registered with the National Ethics Committee. All the participants provided written informed consent.

\section{Stimuli}

Stimulus materials were generated by taking ecological pictures of social gestures. Four semiprofessional actors ( 2 males and 2 females) were asked to spontaneously display gestures using their entire body (emblems, deictic, iconic gestures). Photographs were taken in a classroom while the actors stood in front of the camera in a light-controlled conditions. A set of standardized instructions was given to each actor, indicating that they should spontaneously express 42 social gestures.

For each of the 42 gesture categories, 4 pictures were taken, which resulted in a total of 168 pictures. Half of these pictures were assigned to the congruent condition, and the other half was assigned to the incongruent condition (Fig.1). For each picture, we associated an auditoryverbal expression that could be congruent or incongruent with the displayed gesture.

Two female and two male native Portuguese speakers produced the auditory-verbal expressions (mean duration $=1088 \mathrm{~ms}, \mathrm{SD}=305 \mathrm{~ms}$ ), which were recorded in a single session in a sound-attenuating booth. The sentences were standardized for sound quality using the normalize function of the audio software Ocenaudio v. $3.3 .10(44.1 \mathrm{kHz}, 16$ bit, stereo).

In the congruent condition, the pictures were congruent with verbal descriptions, while in the incongruent condition, the pictures were incongruent with the verbal descriptions. Stimuli were balanced so that each actor appeared in an equal number of congruent and incongruent stimuli. 
To obtain an optimal temporal synchronization between EEG/ERP recordings and brain processing of gestures, images were flashed as static pictures (not as dynamic short movies).

To test the validity of the pictures (i.e., to ensure that they were easily comprehensible in terms of their intended meanings), they were presented to a group of 10 judges of similar age (mean age $=24.3, \mathrm{SE}=2.25)$ and educational levels as the experimental subjects. These judges were asked to judge the coherence between the gesture of the pictures and the verbal labels.

Congruent stimuli that were rated as incoherent by more than $20 \%$ of judges were discarded, as were incongruent sequences evaluated as coherent. Our final stimulus set included 84 stimuli per condition. A total of two lists (each consisting of 168 stimuli in random order) were created, with the two conditions counterbalanced such that participants viewed each stimulus only once in a list.

\section{Congruent}

\section{"Look at that!"}
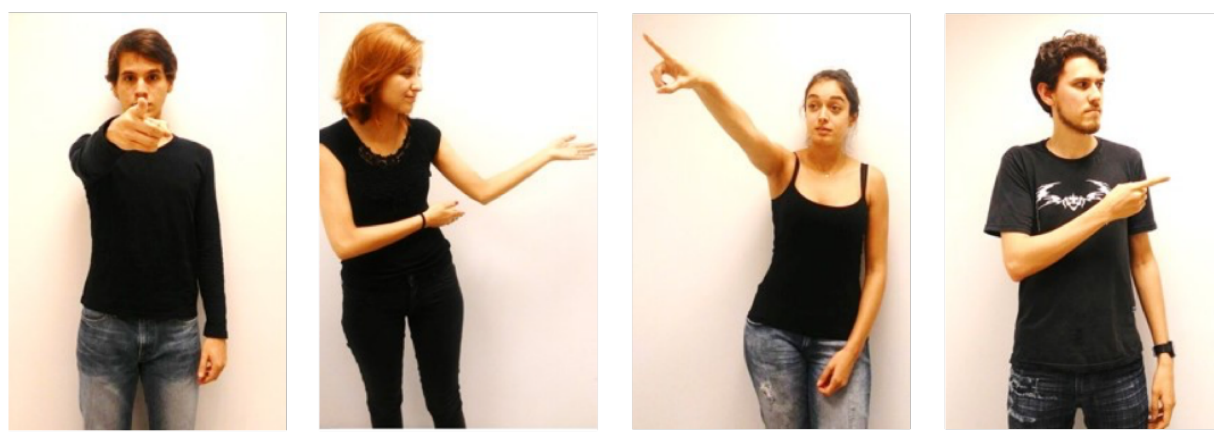

\section{Incongruent}

"I don't like you!"
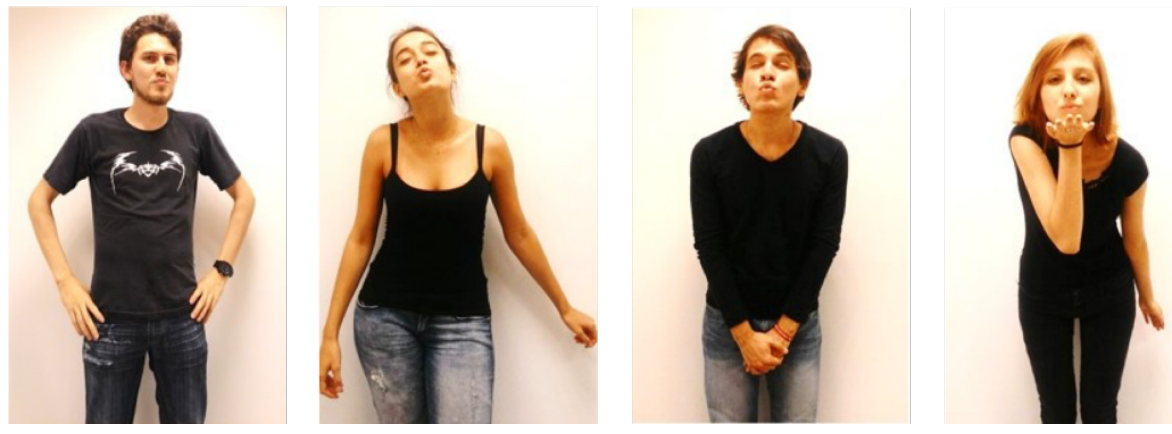
Figure 1. Examples of the body gestures portrayed by the male and female actors in the congruent (above) and incongruent conditions (below).

\section{Procedure}

The task consisted of responding as accurately and quickly as possible to the presence of landscapes or interior (scenarios without visible persons) by pressing a response key with the index finger of the left or right hand while ignoring all other pictures. The two hands were used alternately during the recording session. The order of the hand and task conditions was counterbalanced across subjects.

Participants sat in front of a monitor in a soundproof, electrically shielded recording chamber. Before each picture, a fixation cross appeared for a duration of $1500 \mathrm{~ms}$. The pictures were presented at the center of the screen, and the auditory sentence was time-locked to each picture. Pictures and verbal expressions were presented for 3s, separated by an ISI of $1000 \mathrm{~ms}$.

Participants were instructed not to blink or move during the experimental session. The experiment had five sections separated by breaks. Experimental trials were preceded by a short practice to familiarize participants with the procedures.

The order of presentation of the stimuli was randomized before the beginning of each recording session.

\section{EEG recording parameters}

The electroencephalogram (EEG) was recorded from 128 electrodes at a sampling rate of 250 $\mathrm{Hz}$ (bandpass $0.01-100 \mathrm{~Hz}$ ). The EEG was recorded and analyzed using the Net station software (Geodesic EEG Net Station, EGI, Eugene, OR). The impedance of all electrodes was kept below $50 \mathrm{k} \Omega$ over the experiment. All recordings were referenced to $\mathrm{Cz}$ electrode during data acquisition. This solution allowed us to analyze the mastoid-temporal lobe activity in 
addition to all other important sites for linguistic processing. EEG epochs were synchronized with the onset of stimuli presentation (Fig.2).

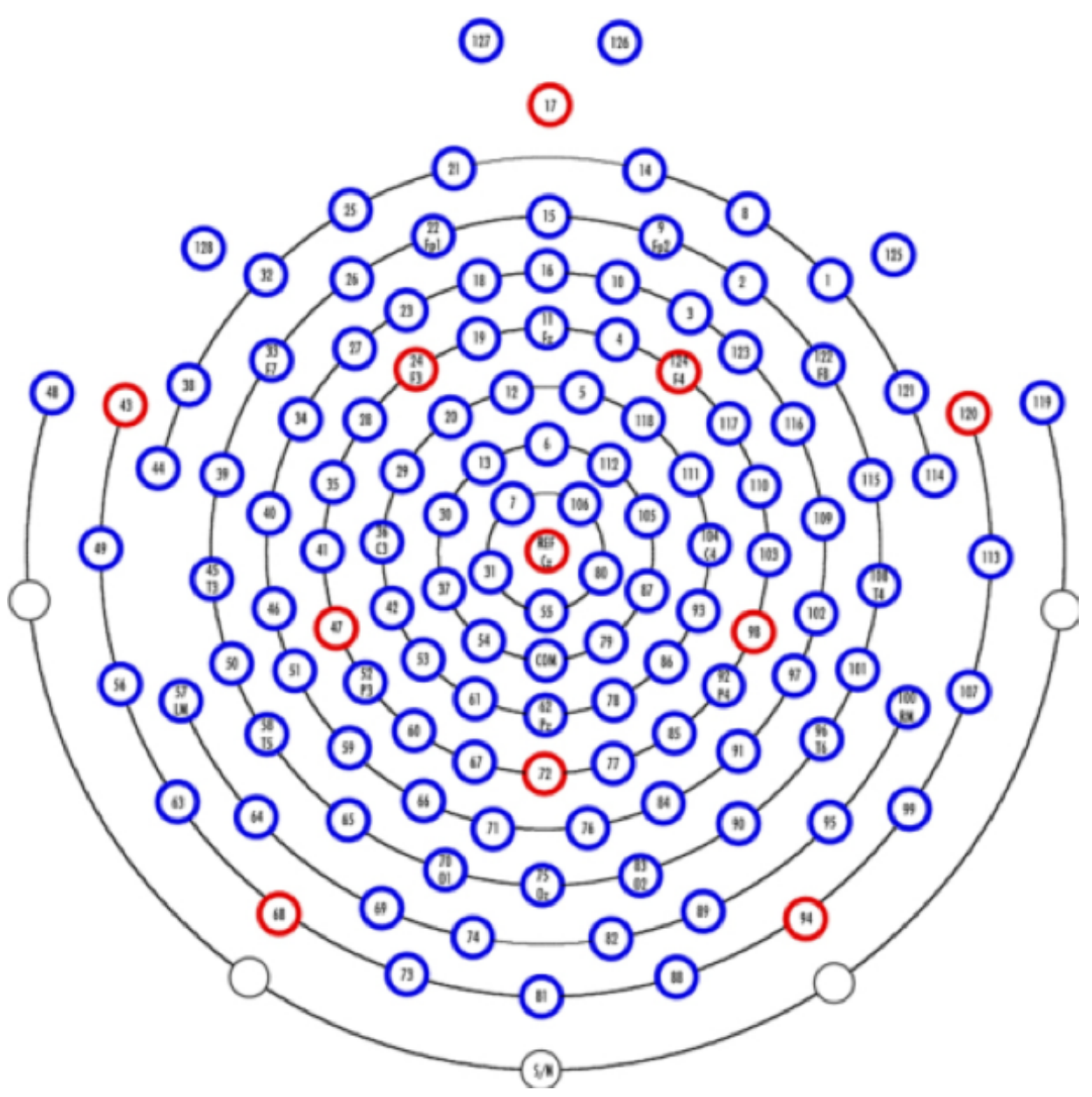

Figure 2. Schematic showing the 128-channel array of scalp electrodes from which the EEG was recorded.

\section{Statistical analysis of ERP responses}

Trials contaminated by blinks, muscle tension (EMG), channel drift, and/or amplifier blocking were discarded before averaging. Approximately $9 \%$ of critical panel epochs were rejected due to such artifacts, with losses distributed approximately evenly across the four conditions. Each participant's EEG was time-locked to the onset of critical panels and their accompanying 
auditory stimuli, and ERPs were computed for epochs extending from $100 \mathrm{~ms}$ before stimulus onset to $1500 \mathrm{~ms}$ after stimulus onset.

Our analysis focused on three epochs of interest. We investigated the mean amplitude voltage and latency of the N2 in the $150-350 \mathrm{~ms}$ epoch, the N400 in the $350-550 \mathrm{~ms}$ epoch and of the LP in the 550-750 ms epoch. These responses were measured at 42 electrode sites (14 in each region) in frontal $(18,16,10,19,11,4,22,23,9,3,124,24,21,14)$, central $(13,6,112$, $7,106,31,80,55,30,105,87,79,54,37)$ and posterior $(61,62,78,67,72,77,71,76,75,81$, $70,83,82,74)$ regions.

The mean amplitude of each component was analyzed using repeated-measures ANOVAs with one factor between (Sex: males, females) and three factors within groups. The within factors were: Congruency (2 levels: Congruent, Incongruent), Region (frontal, central, posterior), Electrode (14 levels). Multiple comparisons of means were performed with posthoc Fisher's tests.

\section{Results}

\section{Electrophysiological results}

N2 (150-350 ms)

The ANOVA performed on N2 mean area yielded significance for the Region factor (F 2, 42 $=38.19 ; \mathrm{p}<0.01)$, revealing smaller responses in the posterior areas $(3.86 \mu \mathrm{V} ; \mathrm{SE}=0.55)$ compared to frontal $(-0.69 \mu \mathrm{V} ; \mathrm{SE}=0.36)$ and central ones $(-0.02 \mu \mathrm{V} ; \mathrm{SE}=0.31)$.

Latency. The ANOVA showed a main effect of Region (F 2, $42=26.03 ; \mathrm{p}<0.01$ ), revealing slower $\mathrm{N} 2$ response in the posterior sites $(273 \mathrm{~ms}, \mathrm{SE}=0.30)$ compared to the frontal $(245 \mathrm{~ms}, \mathrm{SE}=0.31)$ and central $(239 \mathrm{~ms} \mathrm{SE}=0.34)$ ones. The ANOVA also showed a significant a Sex x Congruency interaction $(\mathrm{F} 1,21=5.55 ; \mathrm{p}<0.05)$ with faster N2 latency to Incongruent than Congruent stimuli in females (Congruent: $252 \mathrm{~ms}, \mathrm{SE}=0.35$; Incongruent: 
245, $\mathrm{SE}=0.31)$ than in males (Congruent: $254 \mathrm{~ms}, \mathrm{SE}=0.35$; Incongruent: 257 ; $\mathrm{SE}=0.35$ )

(Fig.3 and Fig.4).

\section{Females}
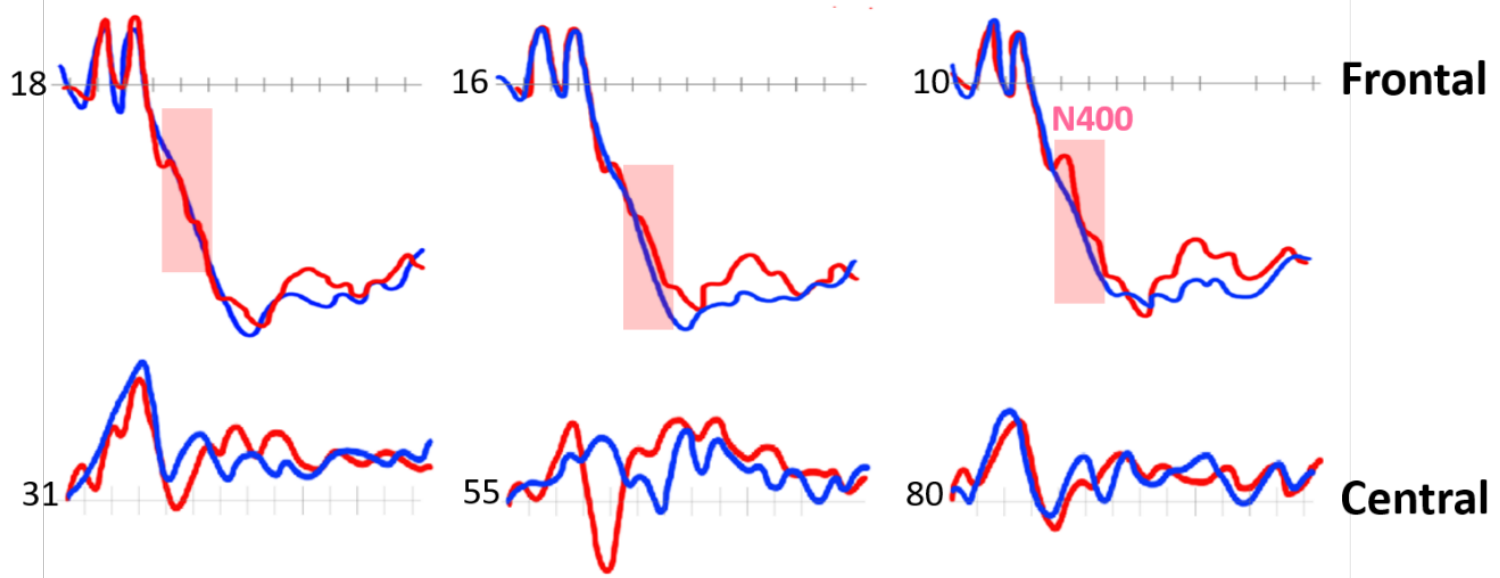

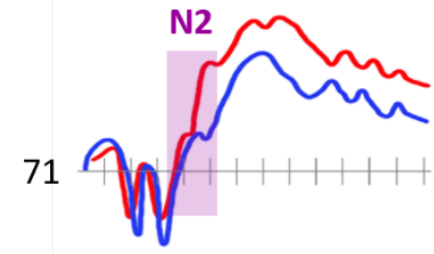

Left

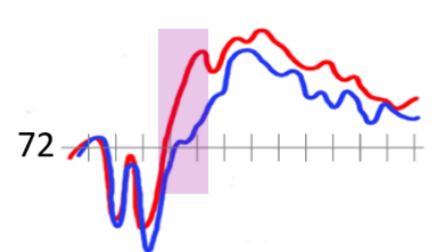

Central

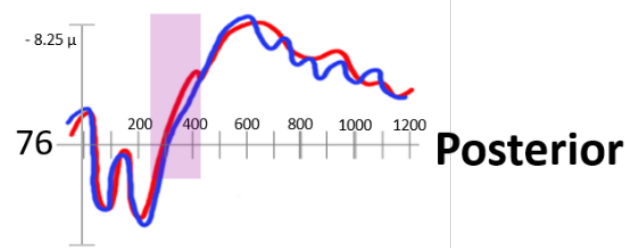

Right

Congruent

Incongruent

Fig. 3. Grand-average ERP waveforms recorded at the frontal, central and posterior electrode sites in response to Congruent and Incongruent stimuli in women. 


\section{Males}
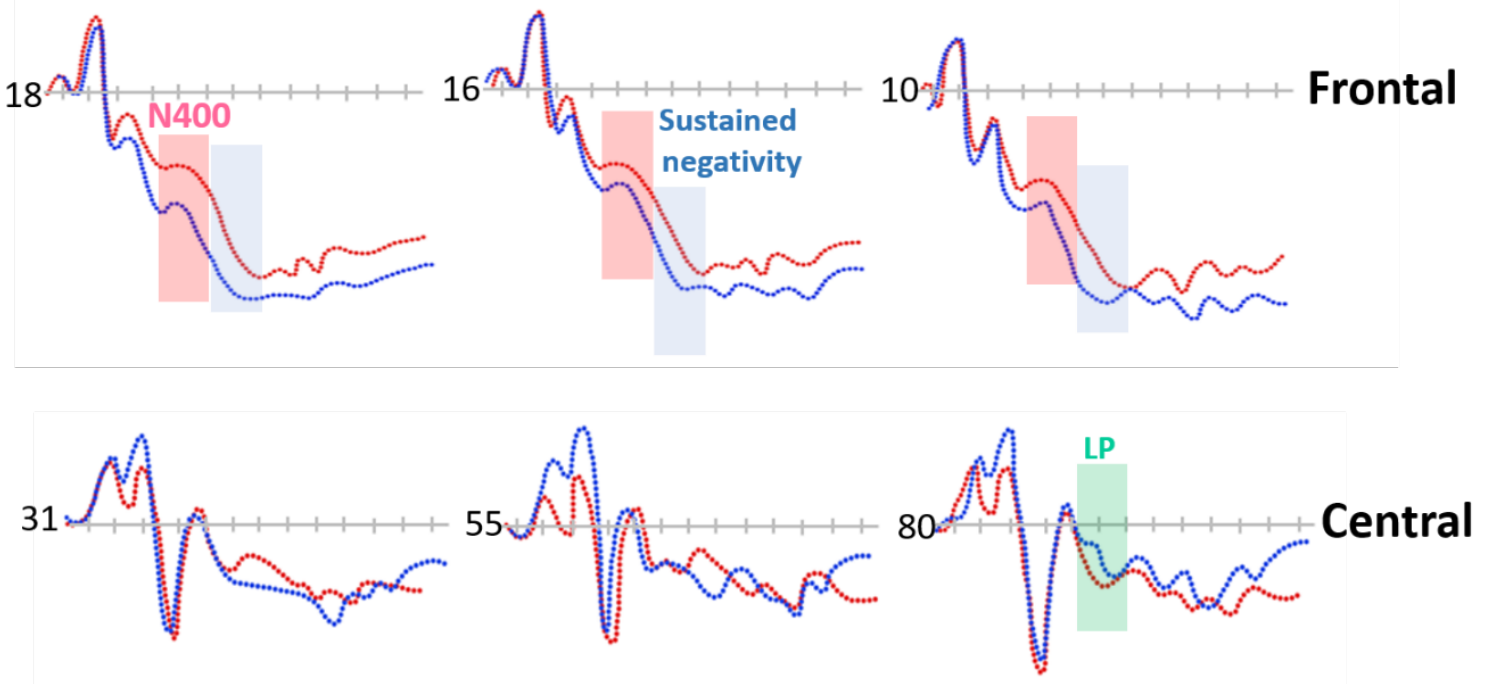

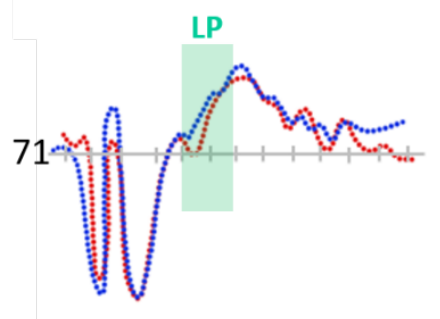

Left

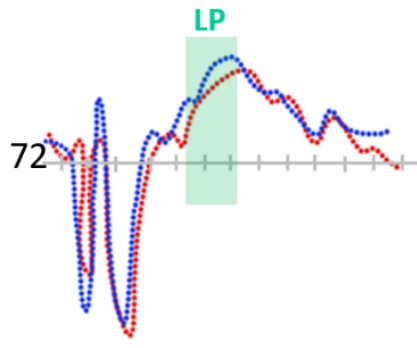

Central

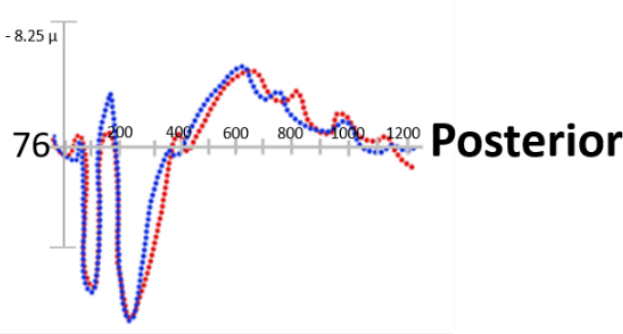

Right

Congruent

Fig. 4. Grand-average ERP waveforms recorded at the frontal, central and posterior electrode sites in response to Congruent and Incongruent stimuli in men.

N400 (350-550 ms)

The ANOVA across regions for the mean amplitude of the N400 component showed a main effect of Region (F 2, $42=6.09 ; \mathrm{p}<0.01)$. The post-hoc analysis revealed a greater negativity in the frontal $(0.15 \mu \mathrm{V} ; \mathrm{SE}=0.43)$ and central sites $(0.70 \mu \mathrm{V} ; \mathrm{SE}=0.37)$ compared to parietal $(1.98 \mu \mathrm{V} ; \mathrm{SE}=0.47)$ ones.

A Congruency $x$ Region interaction (F 2, $42=4.34 ; p<0.05)$ showed that the N400 amplitude was more negative in response to incongruent stimuli than congruent stimuli only in the frontal area (Congruent: $0.35 \mu \mathrm{V} ; \mathrm{SE}=0.39$; Incongruent: $-0.04 \mu \mathrm{V} ; \mathrm{SE}=0.49 ; \mathrm{p}<0.05$ ) 
(Fig.3 and Fig.4). No differences were found between the N400 response to congruent and incongruent stimuli in the central and parietal sites (all $p \mathrm{~s}>0.1$ ). Finally, no differences were found between men and women at any scalp sites (all $p \mathrm{~s}>0.1$ ).

\section{Later effects $(550-750 \mathrm{~ms})$}

The ANOVA computed on the mean amplitude in the later time window, revealed a main effect of Region (F 2, $42=4.85 ; \mathrm{p}<0.05)$, showing that the frontal sites $(2.03 \mu \mathrm{V}, \mathrm{SE}=0.60)$ were significant more positive than the central and posterior ones (Central: $0.74 \mu \mathrm{V}, \mathrm{SE}=0.33$; Posterior: $0.19 \mu \mathrm{V}, \mathrm{SE}=0.31 ; p<0.01)$.

A significant interaction between Congruency, Region and Sex $(F$ 3, 42 $=3.37, p<0.05)$ revealed that incongruent stimuli elicited ERPs that were more negative-going compared to congruent ones over frontal areas only in males group $(\mathrm{p}<0.01$; Incongruent $=1.44 \mu \mathrm{V}, \mathrm{SE}=$ 0.89; Congruent $=2.05, \mathrm{SE}=0.80)($ Fig.3 and Fig.4). Conversely, ERPs to the incongruent stimuli were significantly more positive-going than to the congruent one strips $(p<0.01)$ over the posterior areas in the males group (Fig.3). No differences were found between Congruent and Incongruent stimuli across brain regions in the females group (Fig.2).

\section{Discussion}

The present study aimed to investigate the semantic processing associated with the comprehension of congruent or incongruent communicative co-speech gestures in men and women. According to our hypothesis, if women are more sensitive to social information, such as body language, we expected to observe different brain responses associated with the presentation of speech and communicative gestures in men and women. In line with this hypothesis, our results indicated that only females showed an earlier a N2 response to incongruent stimuli than congruent ones, while larger sustained negativity in response to 
incongruent stimuli was observed only in males. Besides, incongruent gestures elicited an N400 effect over frontal sites compared to the congruent ones in both groups.

In the time window from 150 to $350 \mathrm{~ms}$, a faster $\mathrm{N} 2$ responsewas observed in response to the incongruent stimuli than to the congruent only in the female group. Previous studies proposed that the $\mathrm{N} 2$ component is sensitive to semantic expectancy and might reflect early semantic processing (Dien et al., 2003; Kelly et al., 2004). In 2004, Kelly and colleagues (2004) examined the brain responses to congruent/incongruent speech and hand gesture audiovisual presentations in a group of fifteen participants. The results revealed that the early components analyzed in this study (i.e., P1-N1, and P2) were sensitive to difficulty in integrating speech and gesture. According to the authors, these results suggested that gestures may create a visuospatial context that successively influences the sensory processing of the linguistic information that follows. The authors concluded that gestures might be integrated with speech since the early stages of language processing (McNeill, 1992).

In light of this evidence, the faster N2 observed to incongruent speech-gesture stimuli in our study would confirm that this early component is sensitive to speech and gesture integration (Kelly et al., 2004). However, in our study, only females showed the N2 response to incongruent speech-gesture stimuli, suggesting an expedited ability to integrated speech into communicative gestures in women compared to men. These data are partially consistent with a previous study (Proverbio et al., 2010) that compared the brain responses to semantically congruent and incongruent actions in men and women. The results showed early processing of the action's purpose in the female brain, with a faster parietal N2 in women than men. In light of this evidence, our findings suggest that gestures are processed differently in men and women and that women are faster at integrating speech into gestures.

In the time window from 350 to $550 \mathrm{~ms}$, we observed a greater N400 response to incongruent stimuli than congruent ones only in the frontal area. Several previous studies 
revealed that semantically inconsistent verbal information with gesture/actions could elicit N400 effects (Cornejo et al., 2009b; Coulson \& Wu, 2005; Holle \& Gunter, 2007; Proverbio et al., 2014a; Proverbio et al., 2015; Wu \& Coulson, 2007). In 2015, Proverbio and colleagues performed an ERP study in which a congruent or incongruent description preceded several pictures of actors showing common Italian gestures. The results indicated that incongruent gestures elicited an anterior N400 response in a group of 14 young adults. In a similar study, Proverbio and colleagues (2014b) compared the neural mechanism subserving the ability to comprehend other people's body language in men and women. They measured the brain activity while the participants were presented with pictures of emotional body language preceded by a semantically congruent/incongruent verbal description. The results revealed that both men and women showed a larger N400 effect to incongruent than congruent stimuli. These results are very similar to those observed in our study and suggest a similar ability to integrate the meaning of verbal description with communicative gestures in men and women in this time window.

Also, in Proverbio's study (2014b) the verbal description was visually presented before the presentation of the picture, whereas in our study, the verbal description was presented in auditory form together with the presentation of the picture. Therefore, these results suggest that perhaps verbal description (both auditory and visually) facilitates the semantic processing of communicative gestures.

Finally, the results of our study revealed a larger late positivity to incongruent speechgesture stimuli than the congruent ones only in the males group. This late positivity is sometimes elicited together with the N400 and has been related to a process of reanalysis of the incongruent situation produced by inconsistent meaning (Ibáñez et al., 2011; Sitnikova et al., 2003). Recent studies have been reported of N400 and LP components elicited by meaningful but non-linguistic stimuli such as objects (Ganis \& Kutas, 2003), pictures 
(Federmeier \& Kutas, 2001; Ganis et al., 1996; McPherson \& Holcomb, 1999), gestures (Kelly et al., 2004; Proverbio \& Riva, 2009; Wu \& Coulson, 2007). In our study, this late positivity response might reflect reanalysis costs triggered by the incongruent speech-gesture pairs. This result suggests that, compared to women, men require additional processing costs (reflected in a P600 effect) to process the semantically incongruent combination of communicative gestures and speech. This would then suggest that men may use a processing strategy oriented towards updating to comprehend speech-gesture interaction than women, implying a greater need for backward-looking reanalysis. One possibility is that this greater updating in men is due to the different ability to process social information in men and women: previous studies revealed that women might show a more advanced ability to interpret facial expression and action intention compared with men (Proverbio, 2017; Proverbio et al., 2007; Thompson and Voyer, 2014).

Nevertheless, in addition to this late positivity, a greater sustained fronto-central negativity appeared in the 550-750 ms time window to incongruent compared to congruent speech-gesture stimuli in men. Similar sustained negativities have been observed following N400s in response to visual anomalies of visual narratives or visual events (Cohn et al., 2012; Manfredi et al., 2018; Manfredi et al., 2020a; Manfredi et al., 2020b; West \& Holcomb, 2002). This negative response may reflect a general cost of further processing the inconsistent interaction between auditory information and gesture (Manfredi et al., 2018).

Overall, these findings suggest that women were able to rapidly recognize and process incongruent audiovisual combinations of communicative gestures and speech (as early as 300 ms) whereas men need additional analysis at the later stages of the process.

One important limitation of this study is that the task employed didn't allow us to differentiate the emotional content of social gestures. A previous study by Sokolov and colleagues (2013) revealed gender differences in recognizing the emotional valence of actions 
and body language. In particular, they showed that males revealed a better performance in recognizing happy actions, whereas females were better in recognition of hostile expressions. Therefore, we are aware that future studies will have to use tasks that will better allow to differentiate the emotional content of social gestures. Moreover, another limitation is the small sample size that reduced the statistical power of our effects.

In conclusion, these findings revealed that men and women use different strategies when integrating speech into communicative gestures and that such differences may arise at different stages of the integrative process.

Acknowledgement: Mirella Manfredi was supported by a FAPESP post-doctoral research grant (2015/00553-5). Paulo S. Boggio is supported by a CNPq research grant (311641/2015$6)$.

Declaration of interests: The authors declare that they have no known competing financial interests or personal relationships that could have appeared to influence the work reported in this paper. 


\section{References}

Anderson, L. C., Bolling, D. Z., Schelinski, S., Coffman, M. C., Pelphrey, K. A., \& Kaiser, M. D. (2013). NeuroImage Sex differences in the development of brain mechanisms for processing biological motion. NeuroImage, 83, 751-760. https://doi.org/10.1016/j.neuroimage.2013.07.040

Barrett, S. E., \& Rugg, M. D. (1990). Event-related potentials and the semantic matching of pictures. Brain and Cognition, 14(2), 201-212. https://doi.org/10.1016/02782626(90)90029-N

Bentin, S., McCarthy, G., \& Wood, C. C. (1985). Event-related potentials, lexical decision and semantic priming. Electroencephalography and Clinical Neurophysiology, 60(4), 343-355. https://doi.org/https://doi.org/10.1016/0013-4694(85)90008-2

Coderre, E. L., Cohn, N., Slipher, S. K., Chernenok, M., Ledoux, K., \& Gordon, B. (2018). Brain and Language Visual and linguistic narrative comprehension in autism spectrum disorders : Neural evidence for modality-independent impairments. Brain and Language, 186(June), 44-59. https://doi.org/10.1016/j.bandl.2018.09.001

Cohn, N. (2012). Explaining I Can t Draw : Parallels between the Structure and Development of Language and Drawing. Human Development, 55(4), 167-192. https://doi.org/10.1159/000341842

Cohn, N., Paczynski, M., Jackendoff, R., Holcomb, P. J., \& Kuperberg, G. R. (2012). (Pea)nuts and bolts of visual narrative: structure and meaning in sequential image comprehension. Cognitive Psychology, 65(1), 1-38. https://doi.org/10.1016/j.cogpsych.2012.01.003

Cornejo, C., Simonetti, F., Ibáñez, A., Aldunate, N., Ceric, F., López, V., \& Núñez, R. E. (2009a). Gesture and metaphor comprehension: electrophysiological evidence of cross- 
modal coordination by audiovisual stimulation. Brain and Cognition, 70(1), 42-52. https://doi.org/10.1016/j.bandc.2008.12.005

Cornejo, C., Simonetti, F., Ibáñez, A., Aldunate, N., Ceric, F., López, V., \& Núñez, R. E. (2009b). Gesture and metaphor comprehension: electrophysiological evidence of crossmodal coordination by audiovisual stimulation. Brain and Cognition, 70(1), 42-52. https://doi.org/10.1016/j.bandc.2008.12.005

Coulson, S., \& Wu, Y. C. (2005). Right hemisphere activation of joke-related information: an event-related brain potential study. Journal of Cognitive Neuroscience, 17(3), 494-506. https://doi.org/10.1162/0898929053279568

Cummings, A., Ceponiene, R., Dick, F., Saygin, A. P., \& Townsend, J. (2008). A developmental ERP study of verbal and non-verbal semantic processing. Brain Research, 1208, 137-149. https://doi.org/10.1016/j.brainres.2008.02.015

Dien, J., Frishkoff, G. A., Cerbone, A., \& Tucker, D. M. (2003). Parametric analysis of event-related potentials in semantic comprehension: Evidence for parallel brain mechanisms. Cognitive Brain Research, 15(2), 137-153. https://doi.org/10.1016/S09266410(02)00147-7

Federmeier, K. D., \& Kutas, M. (2001). Meaning and modality: Influences of context, semantic memory organization, and perceptual predictability on picture processing. Journal of Experimental Psychology: Learning, Memory, and Cognition, 27(1), 202224. https://doi.org/10.1037//0278-7393.27.1.202

Ganis, G, Kutas, M., \& Sereno, M. I. (1996a). The search for "“common sense"”: an electrophysiological study of the comprehension of words and pictures in reading. Journal of Cognitive Neuroscience, 8(2), 89-106.

https://doi.org/10.1162/jocn.1996.8.2.89 
Ganis, G, Kutas, M., \& Sereno, M. I. (1996b). The search for "common sense": an electrophysiological study of the comprehension of words and pictures in reading. Journal of Cognitive Neuroscience, 8(2), 89-106. https://doi.org/10.1162/jocn.1996.8.2.89

Ganis, Giorgio, \& Kutas, M. (2003). An electrophysiological study of scene effects on object identification. Cognitive Brain Research, 16(2), 123-144. https://doi.org/10.1016/S0926-6410(02)00244-6

Holle, H., \& Gunter, T. C. (2007). The role of iconic gestures in speech disambiguation: ERP evidence. Journal of Cognitive Neuroscience, 19(7), 1175-1192. https://doi.org/10.1162/jocn.2007.19.7.1175

Ibáñez, A., Toro, P., Cornejo, C., Urquina, H., Hurquina, H., Manes, F., Weisbrod, M., \& Schröder, J. (2011). High contextual sensitivity of metaphorical expressions and gesture blending: A video event-related potential design. Psychiatry Research, 191(1), 68-75. https://doi.org/10.1016/j.pscychresns.2010.08.008

Kelly, S. D., Kravitz, C., \& Hopkins, M. (2004). Neural correlates of bimodal speech and gesture comprehension. Brain and Language, 89(1), 253-260. https://doi.org/10.1016/S0093-934X(03)00335-3

Kutas, M, \& Hillyard, S. A. (1980). Reading senseless sentences: brain potentials reflect semantic incongruity. Science, 207(4427), 203 LP - 205. https://doi.org/10.1126/science. 7350657

Kutas, Marta, \& Federmeier, K. D. (2011). Thirty years and counting: finding meaning in the N400 component of the event-related brain potential (ERP). Annual Review of Psychology, 62, 621-647. https://doi.org/10.1146/annurev.psych.093008.131123

Liu, B., Wang, Z., Wu, G., \& Meng, X. (2011). Cognitive integration of asynchronous 
natural or non-natural auditory and visual information in videos of real-world events: An event-related potential study. Neuroscience, 180, 181-190.

https://doi.org/10.1016/j.neuroscience.2011.01.066

Manfredi, M., Cohn, N., De Araújo Andreoli, M., \& Boggio, P. S. (2018). Listening beyond seeing: Event-related potentials to audiovisual processing in visual narrative. Brain and Language, 185, 1-8. https://doi.org/10.1016/j.bandl.2018.06.008

Manfredi, M., Cohn, N., Sanchez Mello, P., Fernandez, E., \& Boggio, P. S. (2020). Visual and Verbal Narrative Comprehension in Children and Adolescents with Autism Spectrum Disorders: An ERP Study. Journal of Autism and Developmental Disorders, 0123456789. https://doi.org/10.1007/s10803-020-04374-x

Manfredi, M., Proverbio, A. M., Sanchez Mello de Pinho, P., Ribeiro, B., Comfort, W. E., Murrins Marques, L., \& Boggio, P. S. (2020). Electrophysiological indexes of ToM and non-ToM humor in healthy adults. Experimental Brain Research, 238(4), 789-805. https://doi.org/10.1007/s00221-020-05753-7

McPherson, W. B., \& Holcomb, P. J. (1999). An electrophysiological investigation of semantic priming with pictures of real objects. Psychophysiology, 36(1), 53-65. https://doi.org/10.1017/S0048577299971196

Ozyurek, A. (2014). Hearing and seeing meaning in speech and gesture : insights from brain and behaviour. Philosophical Transactions of the Royal Society B: Biological Sciences, $369(1651)$.

Pavlova, M. A. (2017a). Emotion Science in the Twenty-First Century. Time, Sex, and Behavior in Emotion Science: Over and Above. 8(July), 1-5. https://doi.org/10.3389/fpsyg.2017.01211

Pavlova, M. A. (2017b). Review Sex and Gender Affect the Social Brain : Beyond Simplicity. 
250, 235-250. https://doi.org/10.1002/jnr.23871

Plante, E., Petten, C. V, \& Senkfor, a J. (2000). Electrophysiological dissociation between verbal and nonverbal semantic processing in learning disabled adults.

Neuropsychologia, 38(13), 1669-1684. http://www.ncbi.nlm.nih.gov/pubmed/11099725

Proverbio, A.M., Calbi, M., Manfredi, M., \& Zani, A. (2014a). Audio-visuomotor processing in the musician's brain: an ERP study on professional violinists and clarinetists.

Scientific Reports, 4. https://doi.org/10.1038/srep05866

Proverbio, A.M., Calbi, M., Manfredi, M., \& Zani, A. (2014b). Comprehending body language and mimics: An ERP and neuroimaging study on Italian actors and viewers. PLoS ONE, 9(3). https://doi.org/10.1371/journal.pone.0091294

Proverbio, Alice Mado. (2017). Sex Differences in Social Cognition: The Case of Face Processing. 234(January 2016), 222-234. https://doi.org/10.1002/jnr.23817

Proverbio, Alice Mado, Adorni, R., Zani, A., \& Trestianu, L. (2009). Sex differences in the brain response to affective scenes with or without humans. Neuropsychologia, 47(12), 2374-2388. https://doi.org/10.1016/j.neuropsychologia.2008.10.030

Proverbio, Alice Mado, Gabaro, V., Orlandi, A., \& Zani, A. (2015). Semantic brain areas are involved in gesture comprehension: An electrical neuroimaging study. Brain and Language, 147, 30-40. https://doi.org/10.1016/j.bandl.2015.05.002

Proverbio, Alice Mado, Matarazzo, S., Brignone, V., Del Zotto, M., \& Zani, A. (2007). Processing valence and intensity of infant expressions: the roles of expertise and gender. Scandinavian Journal of Psychology, 48(6), 477-485. https://doi.org/10.1111/j.14679450.2007.00616.x

Proverbio, Alice Mado, \& Riva, F. (2009). RP and N400 ERP components reflect semantic violations in visual processing of human actions. Neuroscience Letters, 459(3), 142- 
146. https://doi.org/10.1016/j.neulet.2009.05.012

Proverbio, Alice Mado, Riva, F., \& Zani, A. (2010). When neurons do not mirror the agent's intentions: sex differences in neural coding of goal-directed actions. Neuropsychologia, 48(5), 1454-1463. https://doi.org/10.1016/j.neuropsychologia.2010.01.015

Puce, A., Epling, J. a, Thompson, J. C., \& Carrick, O. K. (2007). Neural responses elicited to face motion and vocalization pairings. Neuropsychologia, 45(1), 93-106. https://doi.org/10.1016/j.neuropsychologia.2006.04.017

Sitnikova, T., Holcomb, P. J., Kiyonaga, K. A., \& Kuperberg, G. R. (2008). Two Neurocognitive Mechanisms of Semantic Integration during the Comprehension of Visual Real-world Events. 2037-2057.

Sitnikova, T., Kuperberg, G., \& Holcomb, P. J. (2002). Semantic integration in videos of real-world events : An electrophysiological investigation. 40(2003), 160-164.

Sitnikova, T., Kuperberg, G., \& Holcomb, P. J. (2003). Semantic integration in videos of real-world events: an electrophysiological investigation. Psychophysiology, 40(1), 160164. http://www.ncbi.nlm.nih.gov/pubmed/12751813

Sokolov, A. N., Enck, P., Kra, I., \& Kru, S. (2013). Emotion through Locomotion : Gender Impact. 8(11). https://doi.org/10.1371/journal.pone.0081716.g001

Van Berkum, J. J. A., Zwitserlood, P., Hagoort, P., \& Brown, C. M. (2003). When and how do listeners relate a sentence to the wider discourse? Evidence from the N400 effect. Cognitive Brain Research, 17(3), 701-718. https://doi.org/10.1016/S09266410(03)00196-4

West, W. C., \& Holcomb, P. J. (2002). Event-related potentials during discourse-level semantic integration of complex pictures. 13, 363-375. 
Wu, Y. C., \& Coulson, S. (2007). How iconic gestures enhance communication: an ERP study. Brain and Language, 101(3), 234-245.

https://doi.org/10.1016/j.bandl.2006.12.003 